\title{
Gobernanza como contradicción: reflexiones sobre el territorio en la configuración de la gobernanza ambiental
}

\author{
Governance as a contradiction: reflections on the territory in the configuration of environmental \\ governance
}

\author{
Historial del artículo \\ Recibido: \\ 22 de mayo de 2020 \\ Revisado \\ 30 de junio de 2020 \\ Aceptado: \\ 02 de noviembre de 2020
}

\author{
Jorge Olea Peñaloza ${ }^{a}$ \\ a Departamento de Ciencias Ambientales, Universidad Católica de Temuco Chile. Correo electrónico: jolea@uct.cl
}

\section{Palabras clave}

Estado, geografía política, gobernanza, territorio.

\section{Keywords}

Governance, political geography, State, territory.

\section{Resumen}

En este texto se reflexiona en torno a la interacción que existe entre gobernanza y gobernabilidad. Al emerger la gobernanza como un aspecto que deriva desde la gobernabilidad -como una profundización y externalización de esta-, señalamos que la gobernanza necesita para su construcción la acción de los sujetos y las instituciones, las mismas que lo constituían previamente. La idea central es mostrar la posibilidad de establecer una reflexión política de la gobernanza a través de la discusión geográfica sobre el territorio. En ese sentido, lo que va transformándose son las relaciones que se establecen entre estos agentes y por lo tanto ahí debiese radicar su especificidad y, por lo tanto, la contradicción entre gobernabilidad y gobernanza debiese estar situada en quienes la ejecutan, así como en las estructuras que emergen de dicha acción. El caso de la gobernanza ambiental nos permite observar que el problema radica en las tensiones entre la gobernanza y los sistemas políticos, así como identificar la especificidad de los agentes involucrados en su funcionamiento. En definitiva, se sostiene que la gobernanza se construye a partir de las experiencias de diferenciación de la gobernabilidad, lo que se logra a través de un análisis territorial, de su materialización a través de los diversos procesos que territorializan las prácticas sociales

\begin{abstract}
This text reflects on the interaction that exists between governance and governability. When governance emerges as an aspect that derives from governability -as a deepening and externalization of this-, we point out that governance needs, for its construction, the action of subjects and institutions, the same ones that previously constituted it. The central idea is to show the possibility of establishing a political reflection on governance through the geographical discussion on the territory. In this sense, what is transforming are the relationships that are established between these agents and therefore there should lie their specificity and therefore, the contradiction between governability and governance should be located in those who execute it, as well as in the structures that emerge from said action. The case of environmental governance allows us to observe that the problem lies in the tensions between governance and political systems, as well as to identify the specificity of the agents involved in their operation. In short, it is argued that governance is built from experiences of differentiation of governance, which is achieved through a territorial analysis, its materialization through the various processes that territorialize social practices.
\end{abstract}




\section{Introducción}

Los conceptos y sus usos siempre están estrechamente ligados a las realidades de las cuales emergen. En este caso, la gobernanza surge como una respuesta a las reestructuraciones de los sistemas políticos y administrativos que surgieron en la década de los ochenta y noventa (Arrighi, 2000). En líneas generales, se pasó de un escenario donde el Estado era el promotor del bienestar de los pueblos, a la necesidad de integrar a la sociedad civil y los sectores privados en la toma de decisiones. Esta transición significó ajustes en varios sentidos y, dependiendo del lugar analizado, tuvo consecuencias que van desde una mayor participación en la toma de decisiones de los grupos que no estaban en la administración del Estado, por ejemplo, las participaciones ciudadanas como requisito para la ejecución de leyes sociales o ambientales, hasta por otra parte, la minimización del Estado, en manos de la sociedad civil y la empresa privada, en el caso de la privatización de la gestión de los derechos fundamentales, como la salud, la educación o los sistemas sanitarios. En definitiva, van surgiendo nuevas plataformas y dispositivos para los vínculos entre la sociedad y el Estado, en la búsqueda de una mayor y mejor forma de articulación de los diversos agentes sociales, así como de los procesos colectivos de toma de decisiones.

La gobernanza, por lo tanto, ha sido esgrimida como uno de los más importantes aportes a la ciencia política de los últimos años, pues ha logrado un nivel de conceptualización que puede proveer dirección y control para la sociedad y su economía (Peters, 2011). Sin embargo, si bien la necesidad de una buena gobernanza, o por lo menos su importancia, es un acuerdo casi total en la literatura sobre ella, existe una importante discusión sobre los medios sobre el cual se construye su aplicación, en los mecanismos desplegados, así como en los contextos sociales y políticos en que se aplican.

Ha sido entendida desde tres ángulos: como una nueva forma de organización social e interacción política, en cuanto proceso sustentado en las condiciones que ha generado el neoliberalismo y sus consecuencias; como un marco deontológico que señalaría la mejor manera de deliberación y toma de decisiones para los agentes políticos; y en tercer lugar, como un concepto analítico que busca establecer marcos teóricos sobre los cuales investigar las nuevas formas de gobierno que se presentan (Bustos-Gallardo, Lukas, Stamm \& Torre, 2019).

Este trabajo busca discutir la gobernanza como esa práctica política, cuya idea central es reflexionar sobre la potencialidad de la gobernanza pues esta se construiría en torno a la posibilidad de participación de la sociedad civil y privada en relación con el Estado, pero que es, a su vez, su propio límite. Para ello se revisarán los principales fundamentos de la gobernanza y en clave teórica se discutirá sobre sus potencialidades y límites, aterrizándolo a la discusión sobre la gobernanza ambiental en el caso latinoamericano.

La idea es mostrar la posibilidad de establecer una reflexión política de la gobernanza, lo que nos lleva a la discusión geográfica sobre el territorio, relevando críticamente que en los procesos de construcción territorial hay elementos para profundizar en la construcción de la gobernanza, tales como los ámbitos de intervención, las instituciones que la sostienen o el grado de participación de sus agentes.

De este modo, en el artículo se asume la gobernanza como una estrategia, que se desarrolla en distintos niveles teoría y praxis, y que ofrece formas concretas de aplicación en la gestión del territorio. Para ello, se recurre como ejercicio práctico al análisis de la gobernanza en general, para luego revisar el caso de la gobernanza ambiental como caso aplicado. De esta manera, se buscó sistematizar las ideas base de la gobernanza, y luego revisar las especificidades que posee la de tipo ambiental.

\section{La gobernanza y contradicción como punto de partida}

La gobernanza como concepto tiene como característica principal el percibirse desde un ámbito teórico hacia lo práctico, es decir, comienza siendo una discusión respecto a las formas que la política debe asumir en un contexto de cambios y, al mismo tiempo, pensar en sus aplicaciones en los diversos escenarios donde corresponde desenvolverse. La gobernanza surge como una adaptación a un período histórico de retroceso del Estado y un resurgimiento del mercado como regulador de las relaciones sociales (LeviFaur, 2012). En suma, podemos entender a la gobernanza como a una forma de interacción política que va más allá de las formas jerárquicas en las que se venía dando la organización política y administrativa de los territorios. Presenta la necesidad de llegar a acuerdos negociados y de gestión en red.

En ese escenario de emergencia y consolidación de nuevas interacciones entre el Estado y la sociedad, se fue materializando la gobernanza a través de un proceso que significó pasar desde la gobernabilidad, es decir, la capacidad de gobernar el gobierno, a una capacidad extragubernamental que fuese más allá de las instituciones tradicionales del gobierno y, por lo tanto, de la creación 
de nuevos pactos sociales y la institucionalización de aquellas relaciones sociales. La gobernabilidad, es decir, la capacidad de desarrollar el gobierno, de acuerdo a dispositivos tecnocráticos y a mantener los equilibrios políticos dentro de la sociedad, dio paso a este nuevo contexto sociopolítico (Ernst \& Haar, 2019). Estos puntos iniciales, básicos a la hora de establecer las bases de la gobernanza, varían de acuerdo a los contextos en que son aplicados, que es donde podemos comenzar a evidenciar los elementos que componen el territorio: sistemas jurídicos, racionalidades burocráticas e intereses económicos (Scott, 2019).

El Estado delegó funciones en organismos privados o de mercado como parte de un proceso de reducción del gasto público, lo que derivó en un cambio en la naturaleza de los servicios públicos (Massey \& Johnston-Miller, 2016). Esto ha sido parte de la Nueva Gestión Pública, sistema predominante en la gestión del Estado y que apunta a una mayor flexibilidad de las estructuras, y autonomía de las agencias, así como en que el manejo de lo público y lo privado tendrían las mismas características, solo transformándose lo específico de sus objetivos (Bryson, Crosby \& Bloomberg, 2014; Dunleavy \& Hood, 1994; Dunleavy, Margetts, Bastow \& Tinkler, 2006). Esto significó, políticamente, una reconfiguración donde el Estado asume su limitada posibilidad de dar cuenta de sus funciones y comenzar a establecer pactos con los otros sectores de la sociedad para asegurar su funcionamiento.

Ese proceso no ha sido lineal ni definitivo, y es más bien un proceso en permanente transformación (Jessop, 2016). El pacto social de las sociedades capitalistas modernas se establece en la interrelación entre los agentes públicos y privados, divididos en tres grupos: el Estado propiamente tal, el sector empresarial y la sociedad civil. Cada uno de ellos también en constante reacomodo, construyendo herramientas efectivas de participación en la política y en la consolidación de instituciones que sostengan el funcionamiento de la sociedad.

A partir de esa amplitud, la gobernanza ha devenido en una propuesta teórica y práctica respecto a cómo afrontar este nuevo escenario social. No obstante, lo que está en juego es la capacidad de los Estados en mantener su centralidad en la organización de las relaciones sociales dentro de este contexto de restructuración de las instituciones que afectan directamente sus formas tradicionales de ejercer el poder. En otras palabras, la acción del Estado en este ajuste, a causa de la gobernanza, está en no perder su centralidad como actor político clave y mantener su hegemonía en la administración. y ejecución de los intereses colectivos (Pierre \& Peters, 2000).
Este nuevo momento, inaugurado por las reformas al Estado como institución a cargo de la mayoría de las relaciones sociales, con la irrupción de una economía global sostenida por una nueva narrativa empresarial respecto a las relaciones sociales (Boltanski \& Chiapello, 2002). Esa nueva configuración de un Estado con otros objetivos en el plano sociopolítico tuvo como consecuencia el surgimiento de otras formas de colaboración social, formales e informales, las que aparecieron de acuerdo a las especificidades de cada territorio. De esta manera, aparece la oportunidad para democratizar las formas de gobierno (Kooiman, 2004), la gobernanza emerge como una herramienta que podría servir para tales propósitos. Por lo tanto, un elemento a tener en consideración en todo esto es que los Estados pasaron de buscar sistemas de planificación a escala nacional o regional, a la búsqueda de sistemas de gobernanza que implican cambios en el paradigma de qué es y qué hace el Estado, a qué escala territorial se plantearán los desafíos y con qué institucionalidad se afrontarán (De Mattos, 2010). Además, no solo se trata de cambios internos en la estructura estatal, sino que este nuevo escenario mundial viene acompañado de nuevos desafíos que requieren de una institucionalidad diferente. El cambio climático, las crisis ambientales o el aumento de la violencia son cuestiones que superan la escala cualitativa y geográfica del Estado. En ese sentido, es una renovación que involucra a cómo entendemos las formas tradicionales de poder y política, abriéndose a formas más variadas de asumir el origen y la práctica del poder político (Pierre \& Peters, 2000).

En este sentido, habría que prestar atención respecto a los elementos que componen ese salto cualitativo que implica superar la gobernabilidad, cuáles son los que se diferencian para llegar a una efectiva gobernanza. Una de las herramientas básicas de la lógica es el principio de la contradicción, la cual propone que una proposición y su negación no pueden ser verdaderas ni tener el mismo sentido al mismo tiempo. Al emerger la gobernanza como un aspecto que deriva de la gobernabilidad - como una profundización y externalización de esta, y de su vínculo estrecho con el Estado- su definición debe ser lo más específica posible, es decir, algo diferente a la gobernabilidad inicial. Sin embargo, la gobernanza necesita para su construcción de la acción de los sujetos y las instituciones, las mismas que lo constituían previamente. En ese sentido, lo que va transformándose son las relaciones que se establecen entre estos agentes y por lo tanto ahí debiese radicar su especificidad. La contradicción en la gobernanza debiese estar situada en el análisis de sus estrategias, de su organización específica. Es por esto por lo que planteamos como solución, un análisis territorial 
de la gobernanza, de su materialización a través de los diversos procesos que territorializan las prácticas sociales. En otras palabras, la gobernanza se construye a partir de las experiencias de superación de la gobernabilidad, es decir, de una geografía política de su práctica.

\section{La gobernanza como concepto de discusión}

La discusión sobre qué es la gobernanza ya tiene varios años y resultaría difícil realizar una problematización en profundidad respecto al tema en este texto. Una de las discusiones centrales dentro de la gobernanza está contenida en la forma dialéctica, a veces, dialógica en otras, que toma su propia conceptualización. No obstante, podemos plantear que la discusión principal ha estado concentrada en torno a la cuestión de si la gobernanza es un concepto teórico o es más bien un fenómeno en sí que no puede ser delimitado y obedece más a una práctica (Aguilar, 2006). Esto es interesante, pues al avanzar en los espacios más específicos de su discusión interna, la gobernanza se plantea como diversa, compleja y dinámica (Kooiman, 2004), y en la práctica es posible ver, a mi juicio, una polarización respecto a qué es lo que hace: o teoriza o pone en práctica ${ }^{1}$. Esta situación presenta un claro desequilibrio, pues las discusiones exclusivamente teóricas no cobran tanto realce como sí lo hacen los estudios de caso y normativos que ocupan una gran parte de la discusión en lo que respecta a la gobernanza.

Desde un punto de vista teórico, la gobernanza ha sido señalada como la organización de actores independientes envueltos en relaciones complejas de interdependencias, quienes con el objetivo del beneficio mutuo gestionan sus propias contradicciones (Jessop, 2002). Es una "heterarquía", es decir, una coordinación e interrelación entre sistemas y organizaciones en diferentes escalas, las cuales operan en la sociedad junto con las formas jerárquicas (autoridad) y económicas (mercado) de articulación. En el contexto de la gobernanza, la heterárquica sería la que ha ganado mayor terreno (Jessop, 1998). La gobernanza podemos señalar, junto con Klijn \& Koppenjan (2012), como un set de instituciones y actores que funcionan más allá de la frontera de un gobierno determinado, en conjunto a un difuso set de medidas para afrontar las temáticas socioeconómicas, y una interdependencia entre las instituciones y agentes dependientes del Estado, y otro grupo autogestionado. Esto genera dinámicas de poder que apuntan a una acción colectiva, que incluso puede llegar a tener distintos niveles en su organización interna, tanto en su estructura -los distintos agentes e instituciones que están interrelacionados-, como en su operación, en cuanto a los mecanismos utilizados y sus impactos (Jessop, 2009).

La gobernanza, en su práctica, es asumida como los mecanismos y estrategias de coordinación para asumir el desafío de la interdependencia y coordinación entre los agentes autónomos, las organizaciones y sistemas formales de gobernabilidad (Jessop, 2016). A partir de allí surgen diversas combinaciones y niveles en los cuales funciona este dispositivo, para llegar incluso a una coordinación sistemática de las diferentes dimensiones de la gobernanza, lo que ha sido denominado como metagobernanza (Fransen, 2015; Jessop, 2009; Pahl-Wostl, 2019). Por lo tanto, podemos señalar que la búsqueda de los equilibrios entre sus agentes, a través de una distribución del quehacer político, es la línea central de la gobernanza, trayectoria que permite abordar nuestra reflexión.

Esto es posible de asumir a través de dos ámbitos principales -e interrelacionados entre sí- la literatura sobre descripción y la normatividad de la gobernanza. En la primera de ellas, encontramos los textos que apuntan a la gobernanza como una superación de la gobernabilidad en cuanto considera todos los agentes extragubernamentales como puntos críticos de su desempeño, lo que otorgaría una nueva capacidad para ejercer el gobierno (Serna de la Garza, 2010). Se describen las entidades que conforman el sistema, la arquitectura de las relaciones y sus tensiones internas y externas (Kooiman, 2004). Se trataría de una búsqueda de la capacidad de dirección de la sociedad en sus diferentes escalas sociales y espaciales, para apostar por una gobernanza que profundiza en los agentes que no han estado vinculados tradicionalmente al gobierno.

Por su parte, la propuesta normativa apunta a desarrollar una forma apropiada, eficaz y correcta de cómo gobernar una sociedad determinada. Aparecen diferentes propuestas de qué se debería considerar a la hora de armar una buena gobernanza, cuáles pesan más, y cuáles son las potencialidades y limitantes para estructurar una propuesta. Así, los diversos agentes, organizaciones sociales y políticas van definiendo lo que sería un "buen gobierno" o de manera más precisa una "buena gobernanza". En este punto surgen diversas formas de aplicación de los modelos de gobernanzas, los que irán a especializarse tanto en su aspecto temático - gobernanza de los recursos naturales, del agua, de la ciudad, entre otros- $y$, por otra parte, en

${ }^{1}$ Esta cuestión quizás tiene que ver con otro aspecto que no podemos detenernos acá, y es su emergencia en un momento en que las ciencias están determinadas por la fragmentación del conocimiento, y la dificultad de generar conceptualizaciones de mayor escala. 
escalas administrativas -a nivel local, nacional, global, por nombrar las principales-.

\section{La gobernanza entre el programa y la práctica}

Desde el punto de vista temático, una de las principales discusiones está en los cambios relativos al Estado y su relación con la sociedad. Las transformaciones acaecidas a partir de los años setenta a escala global vinieron a reorganizar el mapa del poder político y económico, desarrollándose una forma diferente de capitalismo cuyo motor está en la vuelta a la apertura de los mercados y la configuración de un sistema sustentado ahora en los instrumentos financieros (Harvey, 1988; Jessop, 2016). En ese sentido, la conceptualización sobre gobernanza “surge como respuesta a la 'crisis del gobierno', crisis que presenta un carácter multidimensional (...) Esta mayor dificultad y complejidad del gobierno abre a la necesidad de considerar una mayor pluralidad de actores en la tarea de gobernar" (Delamaza \& Thayer, 2016, p. 139). Sin embargo, por ejemplo en el caso latinoamericano, no fue un cambio directo hacia la gobernanza, sino que comenzó con un cambio que pasó por un rediseño de las organizaciones sociales gubernamentales y extragubernamentales de los Estados impulsadas principalmente por organizaciones multilaterales (Zurbriggen, 2011), para provocar el ajuste de los dispositivos de poder que están dentro del funcionamiento del poder político tradicional (López, 2016).

Esta potencialidad de la gobernanza significó $-\mathrm{y}$ sigue significando- una interesante oportunidad para abordar temáticas que no estaban contempladas en una gobernabilidad tradicional. Una vez puesto en práctica, fueron apareciendo las gobernanzas para "combatir la pobreza", cuestiones como los "desastres naturales", la "gestión de recursos", entre otros, que fueron dando una mayor diversidad a los objetivos que se hizo cargo la gobernanza. Asimismo, estas nuevas temáticas también plantearon el desafío de su escalaridad, pues algunos casos requerían gestiones locales, mientras que otros superaban el espacio nacional, e incluso, requerían un abordaje a escala mundial (Woodhouse \& Muller, 2017).

Esta variedad de enfoques se ha desarrollado en un contexto sociopolítico determinado, es decir, con sus propios niveles y agentes de gobernanza, con profundidades históricas y culturales que en algunas ocasiones han significado abrir el repertorio de posibilidades para el surgimiento de políticas en torno al nuevo escenario político y social. En el caso de América Latina esta cuestión ha llamado la atención de las investigaciones en torno al tema, puesto que los casos analizados muestran por una parte, que son modelos de gobernanza reactivos ante algún problema evidente, y que la óptima interacción entre agentes, en algunos casos presenta desconexión (Bustos-Gallardo, 2013). Asimismo, surgen formas territoriales de gobernanza que deben hacerse cargo de la coordinación de crisis multidimensionales y aunque sus énfasis están en realizar una acción más allá de lo sectorial-económico, actúan más bien como restauradores de la convivencia social (Bustos-Gallardo et al., 2019). Esto podría suponer que las estrategias nominadas como gobernanza han sido definidas en sintonía de los modelos de desarrollo económico que afectaron y afectan los procesos democráticos sobre los que se construye (Riggirozzi \& Wylde, 2018).

En este sentido, comienzan a cobrar importancia las especificidades territoriales desde las cuales se construyen estos procesos de gobernanza. Al comparar los procesos que se dan en Europa y América Latina nos encontramos que en el primer caso se ha hablado generalmente de transformación del Estado, mientras que en Latinoamérica ha sido un caso más de transferencias de formas de hacer la gobernanza (Zurbriggen, 2011). Asimismo, las preocupaciones políticas que influyen los diferentes tipos de énfasis desde la gobernanza han variado desde los conflictos distributivos con base en el mercado en las décadas de los ochenta y noventa, hacia un énfasis en los derechos civiles y socioambientales en las últimas décadas (Riggirozzi \& Wylde, 2018). Incluso esta etapa final se encuentra con que gran parte del continente está en procesos de "retorno del Estado" a través del posneoliberalismo producido por el surgimiento de la "nueva izquierda" (Grugel \& Riggirozzi, 2012). Cada uno de estos períodos y procesos tensionan los procesos globales de gobernanza, con urgencias y contextos que varían con cada sistema político.

Desde el punto de vista normativo, la gobernanza apunta principalmente a la necesidad de un "buen gobierno", que exista en cierta forma una manera apropiada, eficaz y correcta de cómo gobernar una sociedad determinada (Massey \& Johnston-Miller, 2016; Peters, 2011). Nuevamente es necesario tener en cuenta la escalaridad de lo que se pretende regular, pues una normatividad que apele a un tema como el orden económico global requerirá de una institucionalidad muy específica y, por lo tanto, se apelará a mecanismos y agentes que operan en otra escala. Asimismo, considerar las especificidades de cada sistema político en el cual se desenvuelve, sobre todo en cuanto a la necesidad de generar instrumentos que materialicen los propósitos finales de la gobernanza. 


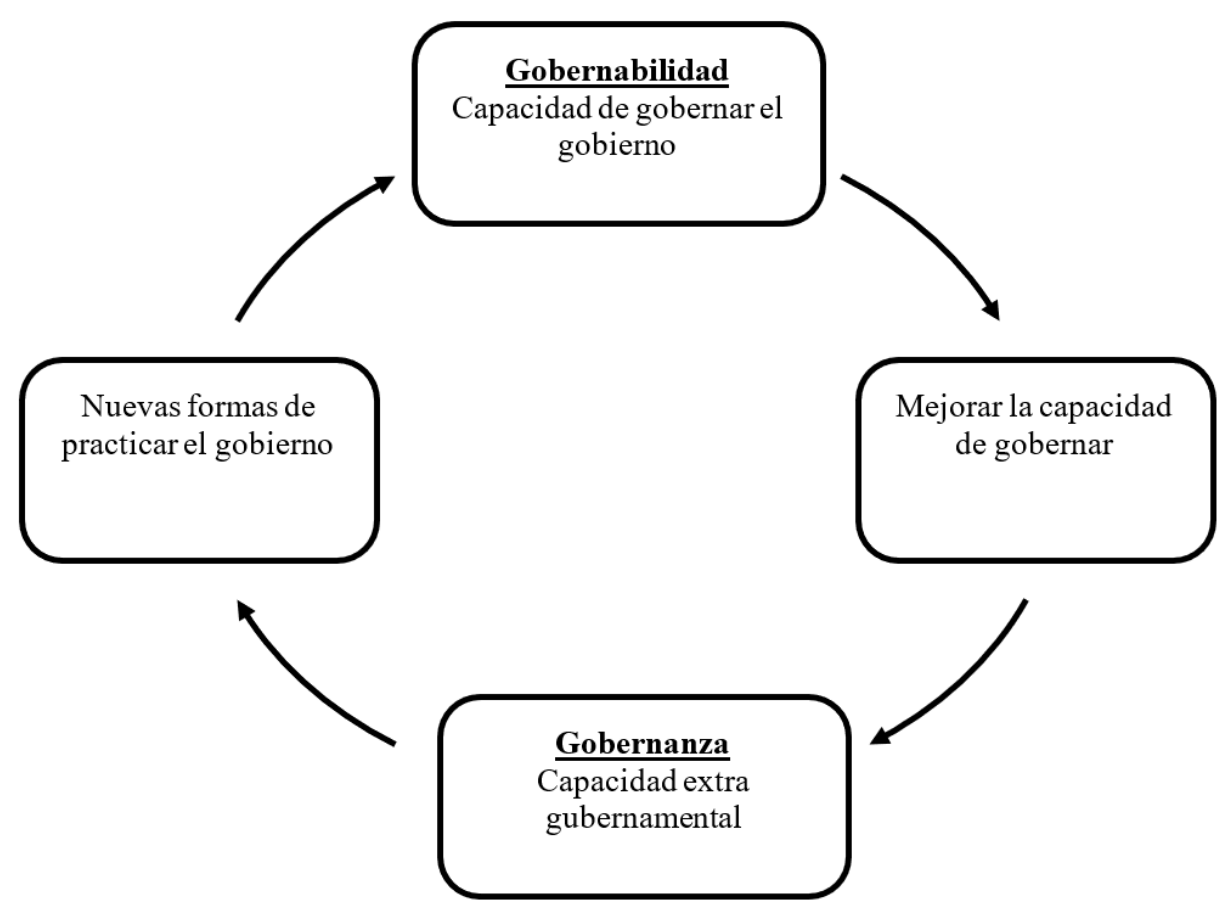

Figura 1. Círculo normativo/prescriptivo de la gobernanza. Fuente: Elaboraciòn propia.

\section{Gobernanza en el territorio}

Para discutir la gobernanza creemos necesario hacerlo desde su funcionamiento. Ese proceso, a su vez, debe ser entendido en relación a su contexto, el que en este caso hemos identificado con el territorio. Al dibujar la forma en que la gobernanza debiese funcionar, nos encontramos con un círculo normativo/prescriptivo que nos muestra como la gobernabilidad es convertida en gobernanza (ver ). Este primer paso permite identificar qué factores impulsan la búsqueda de mejorar la capacidad de gobernar, lo que da lugar a la gobernanza, una estrategia política de rescribir el pacto social sobre el cual se organiza la sociedad. Esto da como resultado nuevas formas de practicar el gobierno, es decir, nuevas tecnologías, instituciones o dispositivos que reorganizan las interacciones entre los agentes. De esta manera, el círculo se cierra con la consolidación de esas nuevas prácticas y estas comienzan a ser parte de una estructura mayor, el gobierno, que nuevamente requerirá ser reforzada y actualizada.

Este modelo ideal generalmente se encuentra con una variedad de dificultades para su concreción. Las condiciones políticas de su representatividad, de las características de su democracia, de los equilibrios entre agentes (Olivo, Alaníz \& Reyes, 2011). A veces se encuentra con que el proceso de gobernanza impulsado es contradictorio con su propio fin, ya que la participación que se promueve va de la mano con los procesos de degradación de los derechos que se están defendiendo. Esto queda muy patente en el caso de las gobernanzas ambientales en Latinoamérica, pues en general se impulsa la participación ciudadana en los casos en que existe una mayor conflictividad socioambiental (de Castro, Hogenboom \& Baud, 2016).

Asimismo, este círculo a veces se rompe al transformarse en una estrategia, en una tecnología de poder por parte del Estado. En general, la gobernanza comienza siendo una propuesta por parte de los agentes para que estos puedan solucionar de la manera más autónoma sus problemas, a través de un sistema de gobierno no jerárquico (Klijn \& Koppenjan, 2012). Sin embargo, termina siendo una estrategia de control social, puesto que se aplica como un proceso de transferencias de modelos externos o de organismos multilaterales (Grugel \& Riggirozzi, 2012), o se ha desplegado como una tensión perpetua entre instituciones políticas débiles que se reproducen socialmente o entre conflictos sobre reconocimiento político, derechos sociales y problemas de distribución del ingreso (Riggirozzi $\&$ Wylde, 2018). Incluso convirtiéndose en un régimen disciplinario que cooptó movimientos potencialmente radicales (Baracco, 2018). 
Esto tiene diversas consecuencias, pues la puesta en práctica de la gobernanza significó la apuesta por la reconfiguración de la política institucional con la inserción de nuevos actores que no estaban considerados. Esto tuvo como una de sus consecuencias principales la disputa por una mayor capacidad de decisión e influencia de los actores no gubernamentales, lo que es refrendado por la práctica de una nueva gobernabilidad, basada en las prácticas y en la acción de gobernar que enfatizan los aspectos que suplen (o, incluso, sustituyen) al poder político de los gobernantes y, a través del Estado, y donde la idea clave es la noción de involucramiento de la sociedad civil con la acción de gobierno.

La gobernanza es un proceso que opera sobre un territorio definido. Al avanzar en una definición de territorio en esa perspectiva, debemos relevar que las especificidades de cada caso están asociadas a procesos sociales que otorgan diferentes demandas a los procesos políticos que reconfiguran los nuevos escenarios surgidos de los programas de gobernanza.

Desde la geografía el territorio ha sido un concepto bastante problemático para utilizar. En una primera lectura, el territorio ha estado tradicionalmente subordinado a la idea de Estado, era el Estado quien delimitaba, controlaba y enunciaba los territorios. Además, era un concepto impreciso, lleno de ambigüedades y retórica, y que solo analizaba el territorio en sí, y no los procesos sociales que este contenía (Santos, 2000, 2005). Ese estrecho vínculo entre territorio y Estado lleva a Lefebvre a reflexionar sobre la tríada Estado, espacio y territorio (Brenner \& Elden, 2009). Se concluye que se consolida un vínculo en la creación de un "espacio estatal", lo que se ha entendido como territorio. Mirado críticamente a través de los postulados de Lefebvre, los autores definen tres puntos para comenzar el análisis: la producción del territorio, las estrategias del espacio estatal y el "efecto territorio", es decir, la tendencia del Estado a naturalizar los efectos de sus transformaciones en las relaciones socioespaciales, a través de sus dispositivos. Al ser concebido el territorio como un espacio estatal, se podría hacer un vínculo con la "producción del espacio" y lograr de esta forma, una "producción del territorio" la cual estaría vinculada al esquema lefebvriano de los espacios percibidos, concebidos y vividos (Magnoli, 2003). En ese sentido, el espacio de la experiencia material, el percibido, estaría asociado a las prácticas territoriales; el espacio de la ordenación y los signos sería el de las disputas por las representaciones del territorio; $y$, por último, el espacio de la imaginación y lo simbólico estaría circunscrito a territorios de representación, todos ellos funcionando en el mismo espacio. Por su parte, Rogério Haesbaert, en su obra El mito de la desterritorialización, hace frente a los análisis que plantean esa externalidad del territorio, así como la superación de este en función de las teorías posestructuralistas y posmodernas que cobran fuerza en las últimas décadas del siglo XX. Según su diagnóstico, estas tendencias teóricas confundirían "la desaparición de los territorios con el simple debilitamiento de la mediación espacial en las relaciones sociales", además, están más preocupadas de visualizar la desaparición de lo antiguo más que del surgimiento de lo nuevo (Haesbaert, 2013).

Para analizar el territorio, por lo tanto, es necesario que el espacio se territorialice, a través de la apropiación. De este modo, se busca entender al territorio como algo que se construye con base en relaciones sociales, el cual se encontraría determinado por las estructuras materiales y los acontecimientos, pero además por sentidos e información (Benedetti, 2011). La propuesta de Raffestin (2013) es que cuando se produce una apropiación de un espacio -ya sea concreta o simbólica- estamos en presencia de una territorialización. A partir de allí, vendrán los procesos de transmutación territorial y de los "múltiples poderes" que actúan en un espacio, y en él se manifiestan múltiples escalas de relaciones de poder. En esa línea el mismo autor plantea la necesidad de deconstruir las relaciones de poder, en tanto la especificidad de las relaciones y de los tipos de poder, pues la territorialización está sustentada en una disputa por el uso y organización del espacio. De este modo, se busca entender al territorio como algo que se construye con base en relaciones sociales, el cual se encontraría determinado en distintos grados por las estructuras materiales y las acciones, pero además por sentidos e información. Esto implicaría que el acceso y el uso de esa información permitiría pensar en los procesos de aparición de nuevos territorios, (territorialización), de destrucción de territorios, que denomina desterritorialización y la reterritorialización, que es una reconstrucción territorial. En síntesis, la propuesta de Raffestin es que cuando se produce una apropiación de un espacio - ya sea concreta o simbólica- estamos en presencia de una territorialización. A partir de allí, vendrán los procesos de transmutación territorial y de los "múltiples poderes" que actúan en un espacio.

La potencialidad analítica del territorio tiene como fortaleza la posibilidad de indagar en distintas escalas, lugares o redes, para poder considerarse de manera conjunta y relacional a todas esas dimensiones, y así lograr una perspectiva mayor de las relaciones socioespaciales en el marco que permita entender las dinámicas que impone el capitalismo contemporáneo, como aparato político, económico y social (Jessop, Brenner \& Jones, 2008). El proceso de 
territorialización va reconfigurando sus gobernanzas y gobernabilidades, para otorgar las especificidades que matizan cada una de ellas. Esto cobra otro sentido cuando observamos el fenómeno desde una escala global, donde los procesos de gobernanza tienen otras demandas y busca articular otros equilibrios de poder.

El cambio climático, las tecnologías de la información, la financiarización de la economía, entre otros, han llamado la atención respecto a la necesidad de afrontar de manera global los problemas que se pueden suscitar. La gobernanza global surge como parte de un nuevo escenario mundial, producto del cambio neoliberal y que tiene consecuencias en el ámbito político internacional y en las relaciones económicas que se desprenden de dichos cambios, cuya consecuencia más relevante es el privilegio del capital y el mercado por sobre el Estado (Jang, McSparren \& Rashchupkina, 2016).

Desde el punto de vista territorial, este escenario y sus "anclajes espaciales" (Harvey, 2005) han sido asumidos por organismos internacionales que han abordado la crisis, tanto en el accionar de los Estados como en las consecuencias del funcionamiento del mercado, que si bien actúan de manera global tiene profundas repercusiones de manera local en el territorio, y donde la gobernanza ofrece diversas alternativas para hacerse cargo (Daher, 2016). En otras palabras, el Estado debe adecuarse a las nuevas territorialidades y sus posibilidades de gobernanza que surgen de los posibles escenarios que surgen desde la globalidad. El traslape en las diversas escalaridades, una global y otra local, otorgan una mayor complejidad a la hora de identificar la mejor manera de encontrar una gobernanza.

En ese sentido, se han levantado algunas críticas a la gobernanza global. Coen \& Pegram plantean que ante el rápido desarrollo de las economías globalizadas y la profundización de la interdependencia de los diversos territorios, la gobernanza no ha podido desarrollar mecanismos efectivos que se hagan realmente cargo de manera global de dichos desafíos (Coen \& Pegram, 2015). Y no es tan solo un problema de escala, sino que de relación entre agentes, pues ante el crecimiento del comercio internacional ha significado una mayor injerencia de los países desarrollados sobre los que están "en vías de desarrollo" (Halabi, 2004). La gobernanza global está relacionada sobre todo a las problemáticas que han surgido en los últimos años -cambio climático, redes digitalesy las no tan nuevas -imperialismo, terrorismo-y que representan nuevos desafíos para mejorar la forma de gobernabilidad y que implica una reconfiguración de los sistemas políticos tradicionales (Sassen, 2003).
El desafío planteado quizás tenga una salida desde una geografía política de dichos procesos, en cuanto las mismas redes sobre las que se teje la estructura global de poder podría revertirse o resignificarse como vías de comunicación para la articulación de los agentes y sus conflictos. Uno de los casos más emblemáticos es la coordinación entre naciones para la defensa de los derechos humanos, el cual implica realzar valores universales, y cuyo desafío no solo queda en manos de una sumatoria de países sino que efectivamente requiere de una heterarquía, en términos de Jessop, para su funcionamiento (Whitman, 2009). Otro ejemplo, es la respuesta al cambio climático, cuya organización no puede ser sino a escala global. Si bien las estrategias de adaptación siguen asumiéndose en una escala nacional, las de mitigación solo pueden ser entendidas en una escala global (Makarov, 2020)

\section{El caso de la gobernanza ambiental}

Una de las formas de gobernanza que ha tomado mayor realce es la gobernanza ambiental. Esta surge en el momento en que el discurso del cambio climático deja de ser un asunto exclusivamente científico y pasa a ser una preocupación de los gobiernos, pasando de ser un discurso técnico a uno tecnocrático. A partir de ese instante, surgirá una institucionalidad que se hará cargo tanto a escala nacional como global de la organización política necesaria para abordar la temática (Taylor, 2013).

Una de las problemáticas más importantes que ha desafiado la gobernanza ambiental en las últimas décadas es la gestión del agua, ante el escenario de creciente escasez. Existe una suerte de trayectoria sobre las preocupaciones en torno al agua que han hecho cambiar paulatinamente la forma en que se entiende su condición en cuanto tal -una suerte de ontología del agua- y al mismo tiempo, las formas en que debiese organizarse las estructuras de gestión y control del agua, las cuales se pueden abordar de manera local y global (Woodhouse \& Muller, 2017). De acuerdo con Fransen (2015), esta gobernanza ha estado últimamente interesada en promover la institucionalización de los actores privados que operan en ella, a través de la regulación por parte de programas de certificación, o en el rol que juegan las organizaciones trasnacionales, a través de arbitrajes comerciales, políticas bilaterales, o el vínculo con otros sectores como la industria de la alimentación o la energía.

Sobre esto ha surgido una serie de propuestas sobre las mejores vías para ejercer una mejor gobernanza, la cual involucra al gobierno, la sociedad y la ciencia, estructurando una gobernanza adaptativa (adaptative 
governance) con énfasis en las transformaciones de los sistemas socioecológicos (Arnold, Gosnell, Benson \& Craig, 2017; Karpouzoglou, Dewulf \& Clark, 2016; Turton et al., 2007). Esto implica entender una gobernanza ya no solo en el recurso en sí, sino en sus componentes territoriales, en las relaciones técnicas y de poder en las cuales se desarrolla su gestión. En otras palabras, se busca incluir en el debate las características ecológicas que intervienen en el recurso, así como en las decisiones e instituciones humanas que regulan su uso.

El cambio en las problemáticas que originan este tipo de gobernanza también hace variar las formas en que se aborda la organización de la gobernabilidad. El caso de las áreas de conservación resulta un ejemplo muy interesante para ahondar en este análisis, pues en el caso chileno, en poco tiempo han reorganizado el mapa de jurisdicción y rol del Estado en la acción de proteger zonas naturales de sus múltiples amenazas, pues si bien reproducen los tres agentes básicos de la gobernanza-Estado, sociedad civil y empresa privada- estos funcionan a una escala tanto local como global (Rivera \& Vallejos-Romero, 2015). El desarrollo de parques privados opera a través de grupos conservacionistas internacionales, los cuales se despliegan sobre el territorio instalando sus propias narrativas de protección del ambiente, lo cual a veces genera conflicto tanto con la legislación vigente o con las comunidades locales (Huiliñir-Curío, Zunino \& De Matheus e Silva, 2019).

Surge la propuesta de avanzar hacia una gobernanza híbrida, que combine propuestas de diseño institucional junto con la autorganización de la sociedad civil (Pahl-Wostl, 2015). El punto crítico de la gobernanza ambiental en el caso latinoamericano es que está vinculado a elementos de supervivencia humana básica (Pahl-Wostl, 2019) así como de ser crítica en territorios marginales del poder central estatal (Composto \& Navarro, 2014). La posibilidad de insertar una gestión coordinada entre los agentes pertinentes sigue estando determinada por la configuración política de los territorios.

Sin embargo, estas gobernanzas ambientales están profundamente vinculadas a los sistemas políticos, que en el caso de América Latina, dependen de sus modelos económicos (Grugel \& Riggirozzi, 2012; Thorp, 1998). Este modelo de desarrollo ha estado fundamentalmente vinculado a la explotación de recursos naturales, lo que ha ubicado a la disputa política en las formas más o menos justas -socialmente hablando- de la distribución de sus ingresos (Delgado, 2013). Esto ha generado una serie de transformaciones del Estado que en la práctica intentan mantener las pautas de crecimiento económico en base a la explotación de la naturaleza.

Las repercusiones que esto tiene en la gobernanza en la actualidad estarían determinadas por ese proceso histórico, que es de larga data y genera un contexto ambiental, social e institucional que empuja a nuevas disputas por el poder político. Más allá de las constantes demandas por injusticias sociales tales como la redistribución por parte del Estado, surgen nuevos movimientos, en base a los agentes que han ido recuperando su presencia política, tales como los y las indígenas o los y las campesinos (de Castro et al., 2016).

Esto presenta como desafío a las gobernanzas el aumento de los conflictos rurales por las amenazas a sus territorios, sobre todo desde la profunda contradicción histórica de América Latina: sectores urbanos demandando mayores recursos los cuales son obtenidos principalmente de la explotación de los recursos naturales, y su consecuente aumento de problemas sobre los territorios en que se encuentran esos recursos (Composto \& Navarro, 2014; Godfrid, 2017; Wagner, 2016). La gobernanza se hace más necesaria en aquellos espacios amenazados y salirse de sus objetivos iniciales - mejorar la gobernabilidad- y transformarse en mediadores de conflictos políticos que superan su capacidad.

Al retomar la idea base del territorio y cruzarla con la discusión sobre la gobernanza, nos surgen dos líneas de reflexión. Primero, el rol del Estado en ambos tiene un lugar central en la configuración de su propuesta teórica y de su reflexión. El Estado sigue siendo el nodo articulador de la gobernanza, independiente de la escala en la que se está mirando el problema, incluso a veces para idear mecanismos que no lo incluyan en su acción. Lo mismo pasa con el territorio, que en su definición inicial requería de una autoridad que delimitara su radio de acción, siendo el espacio el soporte que tradicionalmente se ha escogido para aquello. Segundo, el gobierno en cuanto agente que organiza el Estado cobra un nuevo sentido, pues se promueve una apertura en quienes lo componen, lo que, en consonancia con las propuestas más actuales para estudiar el territorio, nos propone una lectura múltiple de los espacios en que se desenvuelven las sociedades. Esto cobra mucho más sentido si abordamos que esta discusión política se da bajo el contexto del neoliberalismo. Este surge ya sea como una política ideológica o como una cuestión meramente pragmática y, en ambos casos, posee la flexibilidad suficiente como para adaptarse a cualquier escenario (Redden, Phelan \& Baker, 2020). 


\section{Reflexiones finales}

La contradicción, como herramienta lógica, nos habla sobre qué cosas son gobernanza y cuáles no. La literatura nos plantea que son necesarios los actores, sus interdependencias dentro de un sistema complejo, cuyas características institucionales permitan establecer e identificar redes mutuas. Este marco referencial comienza a transformarse y ajustarse de acuerdo con el momento político de cada espacio donde se busca llevar a cabo procesos de gobernanza. En otras palabras, la gobernanza es una tecnología, una estrategia para alcanzar objetivos generales que apuntan a una mejor y más diversa gobernabilidad en los diferentes aspectos que se ocupa el Estado y sus relaciones sociales.

El problema surge cuando esa aproximación al concepto se torna estático o se busca una forma única de gobernanza. La literatura consultada aborda diferentes formas de entenderla y abordarla tanto empírica como teóricamente. En general, se busca llegar a una definición operacional y situada, relevando los aspectos institucionales sobre la cual se construye la gobernanza. La dificultad radica en ver qué magnitud, en cuantificar o cualificar los niveles de gobernanza, así como identificar la especificidad de los agentes involucrados en su funcionamiento.

Un acuerdo entre los textos consultados es la importancia del Estado en la configuración de las redes de la gobernanza. En algunos casos explícito, en otro de manera más indirecta, la referencia a la estructura del Estado está presente en las discusiones. Los inicios de la gobernanza se sitúan en el proceso de transformación del Estado en función de su retroceso como gestor del pacto social entre los agentes y la necesidad de replantear los vínculos políticos que articulan el bienestar social. Asimismo, las demandas siguen siendo hacia el Estado, desde los sectores que necesitan mejoras en sus condiciones -mayormente la sociedad civil, a veces los sectores privados-.

Es por esto por lo que la gobernanza debe entenderse desde una geografía política del Estado. La forma en que comienza a desarrollarse el tejido político de la gobernanza comienza en las instituciones formales que van dando un cierto encuadre a las posibilidades de desarrollo de una mejor gestión del gobierno. No obstante, y siguiendo a Jessop, ese Estado tiene especificidades que, a partir de sus propias trayectorias, van influyendo en dichas posibilidades, pues el que establece las reglas del juego de la gobernanza son las relaciones de poder desde as que se articula el Estado.
El binomio Estado-Gobierno, que tradicionalmente ha marcado la ciencia política, está en el centro de la discusión, y mientras en un una parte importante de los esfuerzos de la gobernanza estaban en potenciar al gobierno, la práctica nos ha demostrado que eso es posible dependiendo de la escala. Sin embargo, la geografía política en América Latina está estrechamente vinculada a los procesos de desarrollo económico y sus formas, al producto democrático -o no-que surge de ahí, y en base a ese resultado, surge el espectro de posibilidad de la gobernanza.

El análisis de la gobernanza en América Latina debería comenzar por un análisis del Estado. Este debe ser un análisis político, del (des)equilibrio de las fuerzas que están ejerciéndose y qué mecanismos permiten mejorarlo o revertirlo si es necesario. La gobernanza ambiental, como caso específico, llama la atención respecto a cómo su presencia está vinculada a procesos de desarticulación política, es decir, a la necesidad de organizar a la sociedad civil en función de los otros dos agentes -Estado y sector privado- o incluso como el caso de Nicaragua de desarticulación intencional de movimientos indígenas. La matriz productiva en base a la explotación de los recursos naturales va generando una serie de conflictos donde la gobernanza, en su idea inicial, requerirá aumentar su impulso y disputar elementos políticos que originalmente no estaban bajo su arbitrio. La gobernanza busca fortalecer instituciones, pero quizás sea el momento de repensarlas desde sus propios objetivos. Si se busca más democracia, estas no vendrán de instituciones poco democráticas

Habría que desarrollar una discusión política sobre gobernanza, puesto que esta sería la forma práctica de entrar a su análisis, a través de su contextualización y de su praxis. Pues, ¿a qué problemas de aplica la gobernanza? A los problemas del desarrollo o, mejor dicho, a los problemas que va dejando el desarrollo. Esto es aún más evidente en el contexto del neoliberalismo, el cual se comporta como una sistema político y económico flexible, lo que también genera que las instituciones se adaptan al sistema, pues son parte de ese "ecosistema neoliberal" que permite la supervivencia del capital y sus gestores. El territorio como configuración social permite evidenciar las maneras en que la gobernanza se asienta en o disputa dichos procesos.

Finalmente, la contradicción sería parte de su propia actividad y consiste en la definición de sí misma y como una vez logrado el paso de la gobernabilidad a la gobernanza este reinicia el ciclo, situación que generalmente toma mucho más tiempo en revelarse o en general una mejor gobernabilidad. El principal riesgo está en que las búsquedas 
iniciales de ese gobierno, tiene la amenaza de convertirse en aquello y, por lo tanto, de institucionalizarse y dejar de ser esa capacidad extragubernamental para afrontar los desafíos, mas, en ese momento preciso debiese aparecer una nueva fuerza que busque una nueva forma de profundizar la gobernanza.

Quizás la gobernanza demora un poco más de lo que debe en reorganizarse como propuesta política y social, o a veces se entrampa en el quehacer de sus prácticas. Esto que no es exclusividad de la gobernanza implica una constante disputa y reflexividad en torno a qué es lo que es y qué debería ser. De esa forma, las contradicciones se materializan en una disputa sobre si debe abocarse a los procesos de gobernanza o a la creación de instituciones, sea cual sea el nivel en el que estemos desarrollándonos. En los trabajos sobre gobernanza, lo descriptivo está principalmente asociado a las técnicas en las cuales se desenvuelve el concepto, mientras que lo normativo está asociado a relaciones de poder, pues quienes puedan participar serán los que estén determinados socialmente para hacerlo, asimismo el cómo, pues dependerá de la estructura social en la cual estén insertas esas prácticas de gobernanza.

Por lo tanto, la cuestión de fondo de la gobernanza está en su relación de poder. En la medida que sea una forma de entender la gobernabilidad, pero a su vez, siga determinada por un gran peso del Estado, seguirá sometida a la relación de poder que es intrínseca a la constitución del Estado. Probablemente, en esa disputa exista una tentación de nominar como gobernanza a algo que aún permanece en una etapa de gobernabilidad. Reconocer esto y hacerse cargo de esa característica permitirá avanzar en las potencialidades directas que tiene la gobernanza, es decir, la democratización de las decisiones en la medida que dichas decisiones equiparen las relaciones de poder de quienes participan en sus determinaciones.

\section{Referencias}

Aguilar, L. (2006). Gobernanza y gestión pública. Ciudad de México: Fondo de Cultura Económica.

Arnold, C. A. T., Gosnell, H., Benson, M. H. \& Craig, R. K. (2017). Cross-interdisciplinary insights into adaptive governance and resilience. Ecology and Society, 22(4). https://doi.org/10.5751/ES-09734220414

Arrighi, G. (2000). Siglo XX: siglo marxista, siglo americano. En VV.AA., Pensamiento crítico contra la dominación. New Left Review. Madrid: Akal.
Baracco, L. (2018). Globalization, Governance, and the Emergence of Indigenous Autonomy Movements in Latin America: The Case of the Caribbean Coast of Nicaragua. Latin American Perspectives, 45(6), 3752. https://doi.org/10.1177/0094582X17705866

Benedetti, A. (2011). Territorio: concepto integrador de la geografía contemporánea. En P. Souto (Ed.), Territorio, lugar, paisaje. Prácticas y conceptos básicos en geografia. Buenos Aires, Argentina: Editorial de la Facultad de Filosofía y Letras, Universidad de Buenos Aires.

Boltanski, L. \& Chiapello, È. (2002). El nuevo espíritu del capitalismo. Madrid: Akal.

Brenner, N. \& Elden, S. (2009). Henri Lefebvre on State , Space, Territory. International Political Sociology, 4(3), 353-377. https://doi.org/10.1111/j.17495687.2009.00081.x

Bryson, J. M., Crosby, B. C. \& Bloomberg, L. (2014). Public value governance: Moving beyond traditional public administration and the new public management. Public Administration Review, 74(4), 445-456. https://doi.org/10.1111/puar.12238

Bustos-Gallardo, B. (2013). The ISA crisis in los lagos chile: A failure of neoliberal environmental governance? Geoforum, 48, 196-206. https://doi. org/10.1016/j.geoforum.2013.04.025

Bustos-Gallardo, B., Lukas, M., Stamm, C. \& Torre, A. (2019). Neoliberalismo y gobernanza territorial: propuestas y reflexiones a partir del caso de Chile. Revista de Geografía Norte Grande, 183(73), 161-183. https://doi.org/10.4067/s071834022019000200161

Coen, D. \& Pegram, T. (2015). Commentary Wanted: A Third Generation of Global Governance Research. Governance: An International Journal of Policy, Administration, and Institutions, 28(4), 417-420. https://doi.org/10.1111/gove.12164

Composto, C. \& Navarro, M. (Eds.). (2014). Territorios en disputa. Despojo capitalista, luchas en defensa de los bienes comunes naturales y alternativas emancipatorias para América Latina. Ciudad de México: Bajo Tierra Ediciones. 
Daher, A. (2016). Externalidades territoriales de la gobernanza financiera global. Eure, 42(126), 213-236. https://doi.org/10.4067/S0250-71612016000200010

de Castro, F., Hogenboom, B. \& Baud, M. (Eds.). (2016). Environmental Governance in Latin America. New York: palgrave macmillan.

De Mattos, C. (2010). Globalización y metamorfosis metropolitana en América Latina. De la ciudad a lo urbano generalizado. Revista de Geografía Norte Grande, (47), 81-104. http://dx.doi.org/10.4067/ S0718-34022010000300005

Delamaza, G. \& Thayer, L. E. (2016). Percepciones políticas y prácticas de participación como instrumento para la gobernanza de los territorios. Un análisis comparado de escalas territoriales en la macrorregión sur de Chile. Eure, 42(127), 137-158. https://doi.org/10.4067/S0250-71612016000300006

Delgado, G. C. (Ed.). (2013). Ecología política del extractivismo en América Latina: casos de resistencia y justicia socioambiental. Buenos Aires: CLACSO.

Dunleavy, P. \& Hood, C. (1994). From old public administration to new public management. Public Money and Management, 14(3), 9-16. https://doi. org/10.1080/09540969409387823

Dunleavy, P., Margetts, H., Bastow, S. \& Tinkler, J. (2006). New public management is dead - Long live digital-era governance. Journal of Public Administration Research and Theory, 16(3), 467494. https://doi.org/10.1093/jopart/mui057

Ernst, R. \& Haar, J. (2019). Globalization, comptetitiveness, and governability. The three disruptive forces of business in the 21st century. Cham: palgrave macmillan.

Fransen, L. (2015). The politics of meta-governance in transnational private sustainability governance. Ecological Economics, 48(3), 293-317. https://doi. org/10.1007/s11077-015-9219-8

Godfrid, J. (2017). Procesos de reconfiguración territorial rural a partir de la implementación de megaproyectos mineros. El caso de La Alumbrera en Argentina. RIVAR, 3(10), 45-69. Recuperado de http://revistarivar.cl/images/vol4-n10/03_Rivar10Godfrid.pdf
Grugel, J. \& Riggirozzi, P. (2012). Post-neoliberalism in Latin America: Rebuilding and Reclaiming the State after Crisis. Development and Change, 43(1), 1-21. https://doi.org/10.1111/j.1467-7660.2011.01746.x

Haesbaert, R. (2013). El mito de la desterritorialización. Del "fin de los territorios" a la multiterritorialidad. Ciudad de México, México: Siglo XXI Editores.

Halabi, Y.(2004). The expansion of global governance into the third world: Altruism, realism, or constructivism? International Studies Review, 6(1), 21-48. https://oi. org/10.1111/j.1079-1760.2004.00371.x

Harvey, D. (1988). Social justice and the city. Oxford: Blackwell Publishing.

Harvey, D. (2005). Espacios de esperanza. Madrid: Akal.

Huiliñir-Curío, V., Zunino, H. M., \& De Matheus e Silva, L. F. (2019). Exclusión y desigualdad en localidades próximas a la Reserva Ecológica Privada Huilo-Huilo en el sur de Chile. Acme, 18(2), 335363. Recuperado de https://acme-journal.org/index. php/acme/article/view/1591

Jang, J., McSparren, J. \& Rashchupkina, Y. (2016). Global governance: present and future. Palgrave Communications, 2, 1-5. https://doi.org/10.1057/ palcomms.2015.45

Jessop, B. (1998). The Rise of Governance and the Risks of Failure: The Case of Economic Development. International Social Science Journal, 50(155), 2945. https://doi.org/10.1111/issj.12186

Jessop, B. (2002). Governance and Metagovernance: On Reflexivity, Requisite Variety, and Requisite Irony. En H. P. Bang (Ed.), Governance, as Social and Political Communication (pp. 142-172). Manchester: Manchester University Press.

Jessop, B. (2009). El futuro del estado capitalista. Madrid: Editorial Catarata.

Jessop, B. (2016). The State. Past, present, future. Cambridge: Polity Press.

Jessop, B., Brenner, N. \& Jones, M. (2008). Theorizing sociospatial relations. Environmental and Plannig D: Society and Space, 26(3), 389-401. https://doi.org/ doi:10.1068/d9107 
Karpouzoglou, T., Dewulf, A. \& Clark, J. (2016). Advancing adaptive governance of socialecological systems through theoretical multiplicity. Environmental Science and Policy, 57, 1-9. https:// doi.org/10.1016/j.envsci.2015.11.011

Klijn, E. H. \& Koppenjan, J. (2012). Governance network theory: Past, present and future. Policy and Politics, 40(4), 587-606. https://doi. org/10.1332/030557312X655431

Kooiman, J. (2004). Gobernar en gobernanza. Revista Instituciones y Desarrollo, 16, 171-195. Recuperado de https://webs.ucm.es/centros/cont/descargas/ documento24211.pdf

Levi-Faur, D. (Ed.). (2012). The Oxford Handbook of Governance. Oxford: Oxford University Press.

López, C. (2016). "Hacer vivir, dejar morir” en la era de la gubernamentalidad. Acerca de la actualidad y de los alcances del enfoque foucaltiano de la biopolítica. Revista de Filosofía, 72, 123-137. http://dx.doi. org/10.4067/S0718-43602016000100008

Magnoli, D. (2003). O Estado em busca do seu Território. Terra Brasilis, (4-5), 1-10. Recuperado de http:// terrabrasilis.revues.org/343

Makarov, I. (2020). Bridging the Gaps in the Polycentric Climate Change Regime. En L. Grigoryev \& A. Pabst (Eds.), Global Governance in Transformation. Challenges for International Cooperation (pp. 163182). Cham: Springer.

Massey, A. \& Johnston-Miller, K. (2016). Governance: Public governance to social innovation? Policy and Politics, 44(4), 663-675. https://doi. org/10.1332/030557314X14042230109592

Olivo, M. Á., Alaníz, C. \& Reyes, L. (2011). Crítica a los conceptos de gobernabilidad y gobernanza. Una discusión con referencia a los consejos escolares de participación social en México. Revista Mexicana de InvestigaciónEducativa, 16(50),775-799. Recuperado de http:/www.scielo.org.mx/scielo.php?script=sci arttext\&pid=S1405-66662011000300006

Pahl-Wostl, C. (2015). Water Governance in the Face of Global Change. From Understanding to Transformation. Cham: Springer.
Pahl-Wostl, C. (2019). The role of governance modes and meta-governance in the transformation towards sustainable water governance. Environmental Science and Policy, 91, 6-16. https://doi.org/10.1016/j. envsci.2018.10.008

Peters, B. G. (2011). Governance as political theory. Critical Policy Studies, 5(1), 63-72. https://doi.org/1 0.1080/19460171.2011.555683

Pierre, J. \& Peters. (2000). Governance, Politics and the State. London: MacMillan Press.

Raffestin, C. (2013). Por una geografía del poder. Michoacán, México: El Colegio de Michoacán.

Redden, G., Phelan, S. \& Baker, C. (2020). Different routes up the same mountain? Neoliberalism in Australia and New Zeland. En S. Dawes \& M. Lenormand (Eds.), Neoliberalism in Context. Governance, Subjectivity and Knowledge. Cham: Palgrave Macmillan.

Riggirozzi, P. \& Wylde, C. (Eds.). (2018). Handbook of South American Governance. New York: Routledge.

Rivera,C.\& Vallejos-Romero,A.(2015).Laprivatización de la conservación en Chile: repensando la gobernanza ambiental. Bosque (Valdivia), 36(1), 15-25. https:// doi.org/10.4067/S0717-92002015000100003

Santos, M. (2000). La naturaleza del espacio. Técnica y tiempo. Razón y emoción. Barcelona: Editorial Ariel.

Santos, M. (2005). O retorno do territorio. OSAL: Observatorio Social de América Latina, 6(16), 251261. Recuperado de http://bibliotecavirtual.clacso.org. ar/ar/libros/osal/osal16/D16Santos.pdf

Sassen, S. (2003). Los espectros de la globalización. Ciudad de México: Fondo de Cultura Económica.

Scott, M. (2019). El "lado oscuro" de la gobernanza del uso de suelo: Las narrativas espacio-temporales y la neutralización del riesgo ambiental. Revista de Geografia Norte Grande, 74, 21-37. https://doi. org/10.4067/S0718-34022019000300021

Serna de la Garza, J. M. (2010). Globalización, Gobernanza y Estado: El caso de la guardería ABC. En H. Fix-Zamudio \& D. Valadés (Eds.), Formación y perspectivas del Estado en México 
(pp. 389-414). Ciudad de México: Universidad Autónoma de México.

Taylor, C. (2013). The Discourses of Climate Change. En T. Cadman (Ed.), Climate Change and Global Policy Regimes (pp. 17-31). London: MacMillan Press.

Thorp, R. (1998). Progreso, pobreza y exclusión. Una historia económica de América Latina en el siglo XX. Washington: Banco Interamericano de Desarrollo.

Turton, A. R., Hatting, H., Maree, G., Roux, D., Claaseen, M. \& Strydom, W. (Eds.). (2007). Governance as a Trialogue: Government-SocietyScience in Transition. Berlin: Springer.

Wagner, L. (2016). Conflictos socioambientales por megaminería en Argentina: apuntes para una reflexión en perspectiva histórica. Areas. Revista Internacional de Ciencias Sociale, 35, 87-99. Recuperado de http:// revistas.um.es/areas/article/view/279201/204181

Whitman, J. (2009). The Fundamentals of Global Governance. New York: Palgrave Macmillan.

Woodhouse, P. \& Muller, M. (2017). Water GovernanceAn Historical Perspective on Current Debates. World Development, 92, 225-241. https://doi.org/10.1016/j. worlddev.2016.11.014

Zurbriggen, C. (2011). Gobernanza: una mirada desde América Latina. Perfiles Latinoamericanos, 19(38), 39-64. Recuperado de http:/www.scielo.org.mx/scielo.php?script=sci arttext\&pid=S0188-76532011000200002 